\title{
Effects of Methylprednisolone on Neuroprotective Effects of Delay Hypothermia on Spinal Cord Injury in Rat
}

\author{
Saeid Karamouzian ${ }^{1,2}$, Sadegh Akhtarshomar ${ }^{3}$, Alireza Saied ${ }^{4}$, Ahmad Gholamhoseinian $^{5}$ \\ ${ }^{1}$ Neuroscience Research Center, Institute of Neuropharmacology, Kerman University of Medical Sciences, Kerman, Iran \\ ${ }^{2}$ Afzal Research Institute (NGO), Kerman, Iran \\ ${ }^{3}$ Neurosurgery Department, Kerman University of Medical Sciences, Kerman, Iran \\ ${ }^{4}$ Department Orthopedics, Dr. Bahonar Hospital, Kerman Neurosceicne Research Center, Kerman, Iran \\ ${ }^{5}$ Department of Biochemistry, School of Medicine, Kerman University of Medical Sciences, Kerman, Iran
}

\begin{abstract}
Study Design: A retrospective study.
Purpose: The aim of this study was to evaluate the effects of delayed hypothermia on spinal cord injuries in rats. In addition, the effect of methylprednisolone on therapeutic window of hypothermia was evaluated.

Overview of Literature: Several studies have demonstrated that early hypothermia is the most effective neuroprotective modality. However, delayed hypothermia seems to be more practical for patients with traumatic spinal cord injuries. A combination of hypothermia and other neuroprotective methods, such as using methylprednisolone, may help extend the therapeutic window of hypothermia. Methods: One hundred and twenty male rats were categorized into six groups. The rats in five groups were subjected to spinal cord injury using the weight drop method, followed by treatment, consisting of early hypothermia, late hypothermia, late hypothermia plus methylprednisolone, or methylprednisolone only. Biochemical tests including catalase, malondialdehyde, and superoxide level were evaluated in the injured spinal cord. Behavioral functions of the hind limb were evaluated by Basso-Battle-Bresnaham locomotor rating scale and tail-flick tests.

Results: Functional and biochemical evaluation showed both early and late hypothermia had significant neuroprotective effects. The treated groups did not differ significantly from one another in the behavioral tests. Hypothermia had better biochemical results compared to methylprednisolone. Also, methylprednisolone was shown to extend the therapeutic window of delayed hypothermia. Conclusions: Hypothermia showed a significant neuroprotective effect, which can be improved with further studies optimizing the duration of hypothermia and the rewarming period. Moreover, the therapeutic effect of the delayed hypothermia can be extended by methylprednisolone.
\end{abstract}

Keywords: Hypothermia; Spinal cord injury; Methylprednisolone; Hypothermia

Received Mar 29, 2014; Revised May 5, 2014; Accepted May 25, 2014

Corresponding author: Saeid Karamouzian

Neurosurgery, Neuroscience Research Center, Institute of Neuropharmacology, Kerman University of Medical Sciences,

Somaie Crossroad, PO Box 113-76175, Kerman, Iran

Tel: +98-9133415316, Fax: +98-3412477925, E-mail: Karamouzian@gmail.com

*The study was supported by Neuroscience Research Center, Institute of Neuropharmacology, Kerman University of Medical Sciences, Kerman, Iran. 


\section{Introduction}

Today, hypothermia is known as one of the strongest neuroprotective modalities. It is commonly used during abdominal aortic aneurysm repair to protect the spinal cord from intraoperative ischemia [1]. However, it has not been used clinically to treat traumatic spinal cord injuries. Patients with traumatic spinal cord injury often arrive at the hospital too late to be candidated for early hypothermia, so an effective delay hypothermia would be more practical. The neuroprotective effect of late onset hypothemia on traumatic spinal cord injuries has not been well determined yet and needs to be studied. Some studies have demonstrated that late onset hypothermia has a neuroprotective effect on ischemic and traumatic brain insults, but this effect was found to be less than that of early onset hypothermia $[2,3]$. The other question that arises is whether the simultaneous use of a neuroprotective agent such as high dose methylprednisolone with late onset hypothermia can enhance or extend the therapeutic window of hypothermia. This study was designed and undertaken to determine whether a neuroprotective agent could extend delayed hypothermia's effective therapeutic window.

\section{Materials and Methods}

\section{Spinal cord injury}

Adult male Wistar rats weighing 250-300 g were used for the study. All the rats were anesthetized with Xylazine $(10 \mathrm{mg} / \mathrm{kg})$ and Ketamine hydrochloride $(60 \mathrm{mg} /$ kg) [4]. Through a dorsal incision, T8-9 laminectomies were performed in the prone position. spinal cord injury (SCI) was induced with the weight drop method. In this method, a stainless steel rod (10 g, 3-mm diameter tip) is dropped through a $2.5 \mathrm{~cm}$ guide tube positioned perpendicularly to the center of the spinal cord [5]. After the surgical and traumatic interventions, the wounds were closed in layers with silk sutures. The Animal Ethics Committee of Neuroscience Research Institute approved the protocols used in this study.

\section{1) Hypothermia}

Hypothermia was induced with a hypothermia machine to keep the rectal temperature at $33.5 \pm 0.5$ degrees [6]. Hypothermia was done via two protocols: early hypo- thermia, in which hypothermia began 30 minutes after spinal cord injury, lasted for 180 minutes, and was followed by a 30 minute rewarming period to slowly bring the temperature back to 37 degrees; late hypothermia, which started 210 minutes after spinal cord injury, lasted for 180 minutes, followed by a rewarming period to slowly bring the temperature back to 37 degrees [7]. The animals were kept under anesthesia during hypothermia in both methods.

\section{2) Experimental groups}

One hundred and twenty rats were randomly and blindly allocated into six groups of twenty rats each: (1) Early hypothermia group: thirty minutes after spinal cord trauma, systemic hypothermia was induced through the early hypothermia protocol. (2) Delayed hypothermia group: three hours after spinal cord trauma, systemic hypothermia was induced through the late hypothermia protocol. (3) Delayed hypothermia+methylprednisolone group: immediately after spinal cord injury, methylprednisolone $30 \mathrm{mg} / \mathrm{kg}[8,9]$ was administered intravenously and followed by delayed hypothermia. (4) Methylprednisolone group: animals received a single high dose of methylprednisolone sodium succinate $(30 \mathrm{mg} / \mathrm{kg})$ intravenously immediately after trauma. (5) Trauma group: animals underwent laminectomy and spinal cord injury. (6) Control group: control animals underwent all surgical procedures except the spinal cord injury.

The animals were allowed access to water and food freely and were kept at the Animal Care Facility at Kerman Medical University Neuroscience Research Center.

\section{Biochemical analysis}

Ten rats from each group were sacrificed 24 hours after spinal cord injury using a lethal dose of ketamine hydrochloride. The operated area was opened, and a $1.5-2 \mathrm{~cm}$ long sample of spinal cord, including the traumatized segment, was obtained. The samples were rinsed with physiologic saline and frozen just after sampling and kept at $-70^{\circ} \mathrm{C}$; malonyldialdehyde (MDA) levels, as an index of lipid peroxidation, were determined by a thiobarbituric acid as detailed by Mihara and Uchiyama [10].

Super oxide dismutase (SOD) level measurements were determined on the basis of SOD's inhibition of the colorimetric reaction due to the superoxide radical [11].

Catalase activity was measured using a colorimetric 
Table 1. Biochemical results in each study group

\begin{tabular}{lcccccc} 
Characteristic & EH & LH & LH+MP & MP & T & C \\
SOD (mmol/mg total protein) & $2.95 \pm 0.83$ & $0.54 \pm 0.53$ & $4.06 \pm 2.03$ & $3.95 \pm 1.08$ & $4.65 \pm 2.72$ & $1.24 \pm 1.44$ \\
CAT (mmol/mg total protein) & $8.40 \pm 3.25$ & $7.45 \pm 2.82$ & $8.95 \pm 5.64$ & $7.70 \pm 2.80$ & $5.54 \pm 3.55$ & $8.44 \pm 2.44$ \\
MDA (nmol/gww) & $54.00 \pm 19.83$ & $59.00 \pm 18.33$ & $47.00 \pm 36.66$ & $74.00 \pm 29.16$ & $92.50 \pm 22$ & $46.00 \pm 22.33$ \\
\hline
\end{tabular}

EH, early hypothermia; LH, late hypothermia; MP, methylprednisolone; T, trauma; C, control; SOD, superoxide dismutase; CAT, catalase; MDA, malondyaldehyde.

assay based on the catalase's dismutation of hydrogen peroxide; absorbance was read in a spectrophotometer at $240 \mathrm{~nm}$ according to the method described by Aebi [12].

Protein concentrations were determined according to Lowry's method [11].

\section{Locomotor function}

Behavioral tests were performed using the Basso-BeattieBresnahan (BBB) scale by an investigator blinded to the treatment groups [4]. Testing began 1 day after the 12.5 $\mathrm{mm}$ spinal cord injury and then continued for six weeks.

\section{Nociceptive function}

Nociceptive function of the spinal cord was evaluated by performing a standardized tail flick test [13]. Each measurement was repeated three times, with at least four minutes between each test.

\section{Statistical analysis}

All the data collected from the experiment were coded, recorded, and analyzed using SPSS ver. 10.0.1 (SPSS Inc., Chicago, IL, USA) software for Windows. Statistical comparisons between the groups were tested with the Kruskal-Wallis test, and the Mann-Whitney $U$ test was used for dual comparisons. In each test, the data were expressed as mean \pm standard deviation, and $p<0.05$ was accepted as statistically significant.

\section{Results}

The MDA level, as an index of lipid peroxidation, was significantly higher $(p=0.006)$ in the trauma group $(92.5 \pm 22$ $\mu \mathrm{M})$ compared to the other groups. As shown in Table 1, hypothermia significantly inhibits MDA. Early hypother- mia kept MDA at a lower level than late hypothermia, a difference which was statically significant $(p=0.004)$. The differences between MDA level in the early hypothermia group and the late hypothermia and methylprednisolone group (LH+MP) was significant ( $p=0.001)$; LH+MP controlled MDA better than just early hypothermia alone. The CAT level was lowest in the trauma group (5.54 \pm 3.55$)$ and the highest in the LH+HP group (8.95 \pm 5.64$)$. Other biochemical findings are shown in Table 1 .

The animals that received treatment exhibited significantly ( $p=0.05$ ) higher BBB locomotor function scores compared with the trauma group 2-3 week after SCI. Importantly, while the animals in the trauma group began to plateau in their locomotor abilities three weeks after injury, the treated animals continued to improve their locomotor abilities for six weeks after injury. There was no significant difference $(p=0.1)$ between the locomotor capabilities of the treated groups. None of the groups achieved full recovery (Fig. 1).

The treated groups all had shorter tail flick latency compared to the trauma group. In the treated groups, the best result was seen in the animals treated with late hypothermia plus methylprednisolone, a difference that was marginally significant $(p=0.054)$.

\section{Discussion}

Many studies have shown the beneficial effect of neuroprotective hypothermia $[8,9]$. In line with these studies, our present study also revealed that early onset hypothermia had a neuroprotective effect, which was not only pronounced in biochemical tests $(p<0.01)$, but also significant in the tail-flick test $(p=0.008)$. Hypothermia works through several mechanisms, one of which is controlling the injury-induced immune response [14]. In this mechanism, hypothermia reduces vasogenic edema and controls chemotaxis of polymorphonuclear leukocytes (PMN) cells [14]. The level of PMN cell activity can be 
measured using the MPO level, which reaches its maximum 3-24 hours after SCI [6]. Hypothermia also delays the glutathione release and reduces the production of O2 free radicals [15]. Meanwhile, it increases the central nervous system's tolerance to ischemia and reduces the anaerobic metabolites [16].

Currently, the only neuroprotective action which is clinically widespread is methylprednisolone prescription [17]. However, in most of the studies, the hypothermia neuroprotective effect was stronger than that of a high dose of methylprednisolone $[4,18]$. In our study, early onset hypothermia had a better neuroprotective effect than methylprednisolone alone. Theearly onset hypothermia group performed slightly better than the methylprednisolone group, but the difference was not significant. However, the tail-flick test in the early onset hypothermia group was marginally $(p=0.054)$ better, and MDA level was significantly ( $p=0.035)$ better than that of the methylprednisolone group. This finding has been also reported by other studies, and it seems hypothermia can play a major role in spinal cord injury treatment going forward.

Some studies on ischemic and traumatic brain injuries have shown that the sooner the hypothermia starts, the better the effect will be $[19,20]$. The only study on the effect of delayed onset hypothermia on SCI found that early onset hypothermia has a better result as compared with hypothermia starting three hours after the injury [21]. In the present study, early onset hypothermia clearly had a stronger neuroprotective effect than delayed hypothermia in the biochemical tests $(p=0.004)$. However, there was not a significant difference between the early and the delayed onset hypothermia groups in the functional tests $(p=0.384)$. The lack of a significant difference in the functional tests between the two groups might have several reasons. Using a non-optimal method of hypothermia is probably the most important reason. Duration and depth of hypothermia and speed of rewarming are factors that influence the results of this method. It has been reported that longer hypothermia has a better effect [22], but the optimal period has not yet been determined. In addition, the length of the rewarming period is important, and using short term rewarming may lead to the return of some of the damaging processes [23]. Nanda et al. [24] have suggested that the rewarming speed of 0.25 degrees centigrade per hour is suitable; in our study, we used a rewarming speed of about 6 degrees centigrade per hour. In the current study, we found that applied hypothermia was more effective on the biochemical tests, possibly because they were checked relatively soon after rewarming. If the duration of hypothermia or rewarming period was not ideal, it is possible the secondary damaging processes started again, which in turn negatively affected the func-

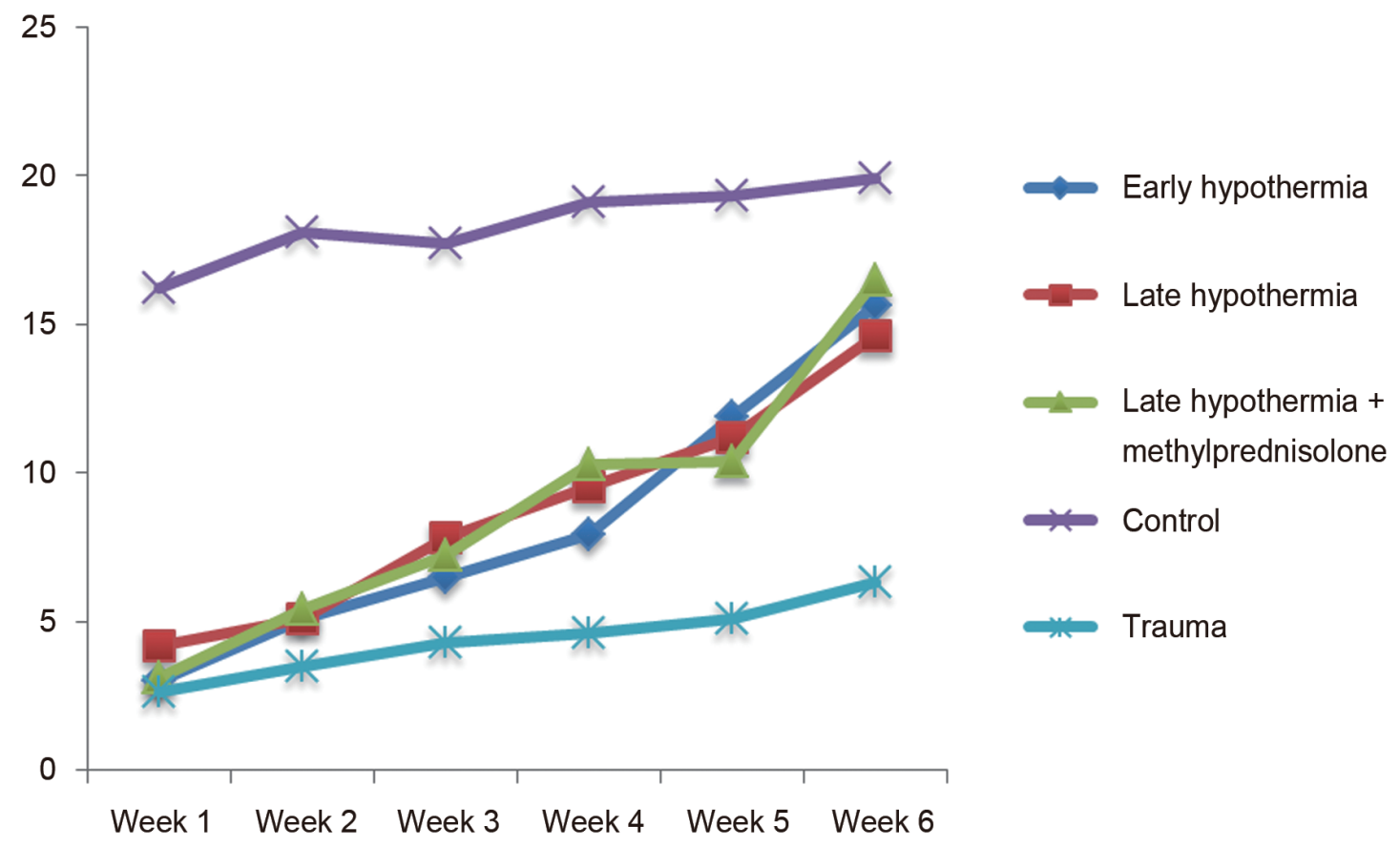

Fig. 1. Basso-Beattie-Bresnahan locomotor function scores results in each study group. 
tional tests because they were checked a relatively longer time after rewarming.

Trauma intensity is another factor that may influence the effects of any neuroprotective treatment [25]. The differences between early and late onset hypothermia methods may be more apparent in different severities of spinal cord injury. The lack of a significant difference between early and late onset hypothermia in functional tests can also be explained differently; it is possible that early onset mechanisms, like calcium hemostasis which starts minutes after injury [26], have less powerful effects on functional disability after spinal cord trauma. Additionally, mechanisms like inflammation [6] and glutathione release or the production of free radicals, which start about three hours after trauma [15], may have more important roles in functional outcome.

Nevertheless, delayed onset hypothermia is more practical in traumatic patients. The transferring and primary stabilization of the patients takes time and makes it difficult to induce hypothermia in the early hospital hours. Combining hypothermia with other neuroprotective actions may intensify the hypothermia neuroprotective effect while also can increasing its therapeutic window. We could only find one other study on the simultaneous use of methylprednisolone and late onset hypothermia [21]. The study found that methylprednisolone did not increase the therapeutic window of moderate systematic hypothermia in mild SCI in rats. On the contrary, in our study, the delayed onset hypothermia with MP had better results in biochemical tests than those of the early onset hypothermia group. This difference was statistically significant $(p=0.001)$. In the study undertaken by Karamouzian et al. [21], the researchers only counted the number of white cells in the injury site to determine the effect of hypothermia, but in our study, we used the myeloperoxidase method, a standard method to examine inflammation [18].

It seems that there is high potential in hypothermia's neuroprotective action and the clinical benefits of delayed hypothermia require researchers to carry out more comprehensive studies to optimize this hypothermia method of treatment.

Our study was limited in that the optimal parameters for hypothermia have not been established, so the results from the present study should be interpreted with caution. However, the result of this study should serve as preliminary results to motivate further research.

\section{Conclusions}

Hypothermia as a neuroprotective modality is more effective compared to MP, and the simultaneous use of hypothermia and MP can increase the effective therapeutic window of hypothermia. However, optimizing the hypothermia method through further study would likely result in a better neuroprotective effect.

\section{Conflict of Interest}

No potential conflict of interest relevant to this article was reported.

\section{Acknowledgments}

We are grateful to Ehsan Mehrabi Kermani (MA TEFL Shahid Bahonar University, Kerman, Iran) for editing the English text.

\section{References}

1. Sundt TM, Flemming MD, Oderich GS, et al. Spinal cord protection during open repair of thoracic and thoracoabdominal aortic aneurysms using profound hypothermia and circulatory arrest. J Am Coll Surg 2011;212:678-83.

2. Urbano LA, Oddo M. Therapeutic hypothermia for traumatic brain injury. Curr Neurol Neurosci Rep 2012;12:580-91.

3. Zhang H, Zhang JJ, Mei YW, Sun SG, Tong ET. Effects of immediate and delayed mild hypothermia on endogenous antioxidant enzymes and energy metabolites following global cerebral ischemia. Chin Med J (Engl) 2011;124:2764-6.

4. Casas CE, Herrera LP, Prusmack C, Ruenes G, Marcillo A, Guest JD. Effects of epidural hypothermic saline infusion on locomotor outcome and tissue preservation after moderate thoracic spinal cord contusion in rats. J Neurosurg Spine 2005;2:308-18.

5. Yu CG, Jimenez O, Marcillo AE, et al. Beneficial effects of modest systemic hypothermia on locomotor function and histopathological damage following contusion-induced spinal cord injury in rats. J Neurosurg 2000;93:85-93.

6. Chatzipanteli K, Yanagawa Y, Marcillo AE, Kraydieh S, Yezierski RP, Dietrich WD. Posttraumatic hy- 
pothermia reduces polymorphonuclear leukocyte accumulation following spinal cord injury in rats. J Neurotrauma 2000;17:321-32.

7. Kuchner EF, Hansebout RR, Pappius HM. Effects of dexamethasone and of local hypothermia on early and late tissue electrolyte changes in experimental spinal cord injury. J Spinal Disord 2000;13:391-8.

8. Dietrich WD. Therapeutic hypothermia for acute severe spinal cord injury: ready to start large clinical trials? Crit Care Med 2012;40:691-2.

9. Kwon BK, Okon E, Hillyer J, et al. A systematic review of non-invasive pharmacologic neuroprotective treatments for acute spinal cord injury. J Neurotrauma 2011;28:1545-88.

10. Mihara M, Uchiyama M. Determination of malonaldehyde precursor in tissues by thiobarbituric acid test. Anal Biochem 1978;86:271-8.

11. Dayan K, Keser A, Konyalioglu S, et al. The effect of hyperbaric oxygen on neuroregeneration following acute thoracic spinal cord injury. Life Sci 2012;90:360-4.

12. Aebi H. Catalase in vitro. Methods Enzymol 1984;105:121-6.

13. Roman JA, Niedzielko TL, Haddon RC, Parpura V, Floyd CL. Single-walled carbon nanotubes chemically functionalized with polyethylene glycol promote tissue repair in a rat model of spinal cord injury. J Neurotrauma 2011;28:2349-62.

14. Lo TP Jr, Cho KS, Garg MS, et al. Systemic hypothermia improves histological and functional outcome after cervical spinal cord contusion in rats. J Comp Neurol 2009;514:433-48.

15. Topuz K, Colak A, Cemil B, et al. Combined hyperbaric oxygen and hypothermia treatment on oxidative stress parameters after spinal cord injury: an experimental study. Arch Med Res 2010;41:506-12.

16. Kakinohana M, Taira Y, Marsala M. The effect of graded postischemic spinal cord hypothermia on neurological outcome and histopathology after transient spinal ischemia in rat. Anesthesiology 1999;90:
789-98.

17. Ko SB, Lee SW. Open reduction without fusion of bilateral jumped lumbosacral facet dislocation associated with symphysis pubis separation and cauda equina syndrome: case report. Asian Spine J 2010;4:128-31.

18. Inamasu J, Nakamura Y, Ichikizaki K. Induced hypothermia in experimental traumatic spinal cord injury: an update. J Neurol Sci 2003;209:55-60.

19. Kumar M, Goldberg AD, Kashiouris M, Keenan L, Rabinstein A. Early hypothermia improves survival and reduces the rise of serum biomarkers after traumatic brain injury in swine. Crit Care 2013;17:P319.

20. Thoresen M, Tooley J, Liu X, et al. Time is brain: starting therapeutic hypothermia within three hours after birth improves motor outcome in asphyxiated newborns. Neonatology 2013;104:228-33.

21. Karamouzian S, Eskandary H, Safizadeh H, Malekpour Afshar R, Adabi S, Gholamhosseinian A. The effect of methylprednisolone on the therapeutic window of systemic hypothermia for the treatment of experimental mild traumatic spinal cord injury in rat. Razi J Med Sci 2009;66:29-35.

22. Mortazavi MM, Verma K, Tubbs RS, Theodore N. Non-pharmacological experimental treatments for spinal cord injury: a review. Childs Nerv Syst 2012; 28:2041-5.

23. Batchelor PE, Kerr NF, Gatt AM, et al. Hypothermia prior to decompression: buying time for treatment of acute spinal cord injury. J Neurotrauma 2010;27: 1357-68.

24. Nanda S, Patel NC, Bhatt SP, et al. Therapeutic medical hypothermia: a multispecialty approach. Recent Pat Cardiovasc Drug Discov 2010;5:212-22.

25. Perlman JM. Summary proceedings from the neurology group on hypoxic-ischemic encephalopathy. Pediatrics 2006;117:S28-33.

26. Levi AD, Casella G, Green BA, et al. Clinical outcomes using modest intravascular hypothermia after acute cervical spinal cord injury. Neurosurgery 2010; 66:670-7. 